\title{
ANÁLISIS EXPLORATORIO ESPACIAL DE TIZÓN COMÚN EXserohilum turcicum (LEONARD AND SUGGS) EN ESTRATOS FOLIARES DE MAİZ, EN VILLA MERCEDES, SAN LUIS
}

\author{
MicCA, M. V. ${ }^{1 ;}$ AndRADA, N. R. ${ }^{2} ; \quad$ \& LARRUSSE, A. S. ${ }^{3}$ (ex aequo)
}

\begin{abstract}
RESUMEN
Los estudios espaciales son necesarios para conocer atributos de contagio de una enfermedad como localización de focos, forma y direccionalidad de un agente causal, y establecer estrategias de manejo. Teniendo como objetivo conocer el efecto del hospedante en la distribución y multiplicación del tizón del maíz Exserohilum turcicum (Leonard and Suggs) se analizaron espacialmente en los diferentes estratos foliares epidemias de tizón en el programa SURFER ${ }^{\circledR}$. Se graficaron las curvas epidémicas y se construyeron mapas geointerpolativos de severidad. El análisis exploratorio espacial de las epidemias demostró el comportamiento acrópeto del tizón del maíz en Villa Mercedes (San Luis). La diseminación de la enfermedad en la planta depende del híbrido, lo que determinará el manejo apropiado para la enfermedad. Es necesario continuar con estudios avanzados, a los efectos de obtener parámetros epidemiológicos temporales y espaciales que permitan comparar epidemias y brindar opciones de manejo adecuadas para la enfermedad.
\end{abstract}

Palabras clave: Distribución espacial, Exserohilum turcicum, mapas geointerpolativos.

\begin{abstract}
Exploratory spatial analysis of corn blight exserohilum turcicum (Leonard and Suggs) in strata leaf of corn, in Villa Mercedes, San Luis.

Spatial studies are necessary to determine the spreading characteristics of disease outbreaks, for example locations of the inoculum source, shape and directionality of a causal agent, and to establish management strategies. To know the effect of the host in the distribution and multiplication of corn "blight", Exserohilum turcicum Leonard and Suggs, they were spatially analyzed in different strata leaf blight epidemics with the SURFER ${ }^{\circledR}$ program. Epidemic curves were plotted and severity geointerpolativos maps were developed. The exploratory spa-
\end{abstract}

1.- Cátedra de Fitopatología. Proyecto 51412. Departamento de Ciencias Agropecuarias. Universidad Nacional de San Luis. Email: nrandrada@gmail.com

2.- Cátedra de Fitopatología. Proyecto 51412. Departamento de Ciencias Agropecuarias (UNSL).

3.- Asesora Externa Proyecto 51412. Departamento de Ciencias Agropecuarias.(UNSL).

Manuscrito recibido el 27 de julio de 2015 y aceptado para su publicación el 5 de abril de 2016. 
M.V. Micca et al.

tial analysis of epidemics showed an acropetal spatial distribution. The spread of the disease on the plant depends on the hybrid, witch will determine the appropriate management for disease. It is necessary to continue with studies that allow to obtain temporal and spatial epidemiological parameters for comparing epidemics and to provide management options for the disease.

Key words: Spatial distribution, Exserohilum turcicum, geointerpolative maps.

\section{INTRODUCCIÓN}

Todos los años en la zona de Villa Mercedes se presenta con distinta intensidad, la enfermedad "tizón común del maíz" Exserohilum turcicum (Leonard and Suggs) (12). Este patógeno, puede afectar a las plantas en cualquier período de su desarrollo; cuanto más temprano se produce el ataque, mayor es el efecto negativo sobre el rendimiento (11). Los primeros síntomas consisten en pequeñas manchas foliares, ligeramente ovaladas y acuosas que luego se transforman en zonas necróticas alargadas y ahusadas. Las manchas se manifiestan primeramente en las hojas basales y en ataques graves, la enfermedad avanza hacia arriba, las hojas se deforman, marchitan y finalmente se secan (10). Cuando se presenta con altos niveles de intensidad, puede provocar madurez anticipada, incompleto llenado de espigas, disminución del peso de los granos, tendencia al quebrado de tallos y posterior vuelco de la planta causado por la removilización de nutrientes del tallo y el consecuente debilitamiento del mismo $(4,6,7)$.

Sillón et al. (18) en monitoreos de enfermedades en maíz en las campañas 2004/052005/06 en la zona centro-oeste de Santa $\mathrm{Fe}$, determinaron que los tizones producidos por Helminthosporium no fueron patologías limitantes, hallándose solo presentes en el 37\% de los híbridos, y su severidad no superó el 10\% del área afectada en la hoja. En el centro-sur de Santa Fe en la campaña 2008/09 se determinaron patologías emergentes con una severidad de tizones entre 0 y $40 \%$ (Exserohilum turcicum y Helminthosporium spp.)(18, 19). Según Couretot et al. $(5,6,7)$ y Formento et al. (10), altos niveles de severidad de tizón generalmente se presentan en maíces de fecha de siembra diciembre/enero en la zona de Paraná, Entre Ríos. En la zona Norte de la provincia de Buenos Aires, en ensayos comparativos de rendimientos y en lotes de producción de maíces de siembra tardía del mes de diciembre, los altos niveles de tizón permitieron caracterizar híbridos con diferente comportamiento. De los híbridos evaluados el $50 \%$ alcanzó niveles de severidad del 45 $60 \%$ mientras que el otro $50 \%$ tuvo niveles entre bajos y moderados del 5 a $25 \%$ (6). En la campaña 2011/12 en maíces de siembra tardía el 57\% de los híbridos presentaron niveles de severidad menores al 1\% y el $43 \%$ de los híbridos restantes alcanzaron valores entre 1,1 y $4 \%$ en $R_{1}$, mientras que en $\mathrm{R}_{4}$ sólo el $26 \%$ de los cultivares pudo conservar niveles de severidad de tizón iguales a $1 \%$ o menores; el $52 \%$ de los híbridos presentó niveles de severidad entre 1,1 y $4 \%$ y el $22 \%$ de los materiales alcanzó severidades mayores al 4,1\% (6). De Ros- 